\title{
Distribuição Espaço-Temporal da Produtividade Média do Café em Minas Gerais: 1997-2006
}

\section{Spatial-Temporal Distribution of the Average Productivity of Coffee in Minas Gerais State: 1997-2006}

\author{
Rudolph Fabiano A. P. Teixeira* \\ Mario Augusto Bertella**
}

\begin{abstract}
Resumo: O presente trabalho tem como objetivo central investigar a distribuição espacial da produtividade média do café entre as 66 microrregiões mineiras durante o período 1997-2006. Pode-se apontar como objetivos secundários: a) analisar a dinâmica espacial; b) identificar a formação de clusters; e c) verificar a incidência de convergência na produtividade média do café, ou seja, testar se a produção de café está se tornando mais homogênea em Minas Gerais. Para isso, implementa-se a análise exploratória de dados espaciais (Aede), bem como a econometria espacial. Conclui-se que a produtividade média do café não segue um processo aleatório, ocorrendo um reordenamento espacial entre as microrregiões. Além disso, verifica-se que essa variável está se distribuindo de forma mais homogênea em Minas Gerais, conforme indicam os modelos de $\beta$ convergência espacial e principalmente o modelo de erro espacial.
\end{abstract}

Palavras-chave: Café. Minas Gerais. Análise exploratória de dados espaciais. Econometria espacial. $\beta$ convergência.

\begin{abstract}
The main purpose of this paper is to investigate the spatial distribution of the average productivity of coffee among the 66 micro regions of the Minas Gerais State between 1997 and 2006. We also have the following objectives: a) to examine the spatial dynamics; b) to identify clusters; c) to check the spatial convergence of the average productivity of coffee, i. e., to test whether the coffee production has become more homogenous in Minas Gerais. In order to do this, we conducted an exploratory analysis of the spatial data (EASD) and used a spatial econometrics as well. We conclude that the average productivity of coffee has not been at random which indicates a spatial reordering among the micro regions. Besides, as the several models show, there has been a spatial convergence concerning the examined variable.
\end{abstract}

Keywords: Coffee. Minas Gerais. Exploratory analysis of the spatial data. Spatial econometrics. $\beta$ convergence.

JEL Classification: C31; R11.

* $\quad$ Mestre em Economia pela FCLAR da Universidade Estadual Paulista Júlio de Mesquita (Unesp). Economista da Eletrobras. E-mail: rudolphgp@hotmail.com

* $\quad$ Doutor em Economia pela UNICAMP. Professor do Programa de Pós-Graduação da FCLAR da Universidade Estadual Paulista Júlio de Mesquita Filho (Unesp). Agradece o apoio do CNPQ sob a forma de Bolsa de Produtividade em Pesquisa. E-mail: mabertella@yahoo.com.br 


\section{1 lntrodução}

O café se destaca na história econômica e social do Brasil desde a época colonial. As primeiras exportações expressivas do produto ocorreram a partir de 1802. Em 1845, o país era responsável por $45 \%$ da produção mundial, destacando-se como o maior produtor. Entre 1925 e 1929, o café chegou a contribuir com $70 \%$ do valor das exportações nacionais. Nos anos de 1950 e 1960, o produto ainda era um dos principais da pauta de exportação brasileira, assegurando receitas cambiais expressivas para a sustentação da política de substituição de importações. Na década de 1970, a produção de café teve um novo impulso com a conquista de regiões dos cerrados, até então pouco exploradas pela agricultura. Muito embora tenha perdido significativa participação no mercado mundial nas duas décadas seguintes, em 1999, com a desvalorização do real, o Brasil voltou a recuperar parcela significativa do mercado internacional de café, elevando sua participação de 20\%, em 1994, para 29,4\% em 2007 (BRASIL, 2009).

Segundo Vale, Teixeira e Sant'anna (2006), a relevância do café pode ser corroborada por diferentes indicadores, tais como: a) o movimento financeiro da cadeia mundial do café; b) o volume de exportações mundiais; c) a capacidade de geração de empregos e; d) a contribuição para o incremento da renda. Além desses, como lembram Souza e Perobelli (2007), a cafeicultura auxilia no desenvolvimento econômico de uma nação por meio da geração de divisas para o processo de industrialização.

No Brasil, o principal estado produtor de café é Minas Gerais, que possui nessa commodity cerca de um terço de seu PIB do agronegócio. Essa cultura é uma das três mais importantes para a pauta de exportações mineiras, ficando atrás apenas do minério de ferro e dos produtos siderúrgicos (BANCO DE DESENVOLVIMENTO DE MINAS GERAIS, 2002).

Assim, analisar o comportamento da produtividade agrícola do setor cafeeiro no estado de Minas Gerais é de suma importância não só para a economia mineira, mas também para a economia brasileira, tendo em vista que o café representa significativa parcela das exportações agrícolas nacionais (em torno 6,6\% na safra 2007, cf. BRASIL, 2009).

Minas Gerais produz dois tipos de café, o arábica (Coffea Arabica L.) e o robusta (Coffea Canephora L.). Entretanto, o estado pode ser caracterizado como um típico produtor do primeiro, já que a produção do último é ainda bastante incipiente, distribuindo-se entre algumas localidades nas mesorregiões Zona da Mata, Vale do Jequitinhonha, Vale do Mucuri, Vale do Rio Doce, Central e Norte (BRASIL, 2009). No ano de 2006, Minas Gerais produziu aproximadamente 30 mil sacas de café do tipo robusta, o que equivale a apenas $0,001 \%$ do total produzido no estado. Em comparação, a produção do café tipo arábica foi de quase 22 mi- 
lhões de sacas no mesmo período, ou 66,5\% da produção nacional (COMPANHIA NACIONAL DE ABASTECIMENTO, 2009).

As condições hídricas e de temperatura são os principais fatores climáticos que influenciam no plantio do café. Déficits hídricos elevados são prejudiciais ao cafeeiro, que, em decorrência, pode apresentar desfolha, secamento dos ramos, morte das raízes e deficiências induzidas de nutrientes. Temperaturas médias anuais entre $18^{\circ} \mathrm{C}$ e $23^{\circ} \mathrm{C}$ são os limites extremos para a cultura, sendo que índices térmicos anuais entre $19^{\circ} \mathrm{C}$ e $21^{\circ} \mathrm{C}$ são os ideais (BRASIL, 2009).

Segundo a mesma fonte, com o uso da irrigação, o café pode ser plantado no estado de Minas Gerais, em qualquer época do ano, porém recomenda-se que seja realizado no início da estação chuvosa, isto é, entre início de novembro e fim de dezembro, época propícia ao "pegamento" e desenvolvimento das mudas.

Devido a sua importância histórica no desenvolvimento do país, além de uma relevante participação no cenário agrícola nacional, o café tem sido objeto de estudo de diversos pesquisadores. Dentre alguns trabalhos recentes, pode-se destacar o de Vale, Teixeira e Sant'anna (2006), que buscam avaliar a eficácia administrativa na produção de café, tomando por base diferentes estilos gerenciais, o de Oliveira et al. (2008), que analisam a estrutura e a dinâmica da cafeicultura mineira através do modelo shift-share, e o de Santos et al. (2009), que investigam o setor de produção e processamento de café em Minas Gerais, utilizando a abordagem da matriz de insumo-produto.

Este estudo tem como objetivo central analisar a distribuição espacial da produtividade média do café, entre todas as microrregiões mineiras (66 no total). Podemos apontar como objetivos adicionais: a) analisar a evolução da dinâmica espacial do café; b) identificar a formação de clusters significativos; e c) verificar a incidência de convergência (ou $\beta$ convergência) espacial na produtividade do café. Afinal, a ideia de utilizar esta análise no trabalho é testar se a produtividade média do café está se tornando mais homogênea entre as microrregiões mineiras com o passar do tempo.

Para que os objetivos delineados neste trabalho possam ser alcançados, será implementada uma análise exploratória de dados espaciais (Aede), responsável por identificar a distribuição espacial, a formação de clusters e a evolução temporal da produtividade cafeeira, além de uma análise de convergência espacial, que busca avaliar se as microrregiões de menor produtividade média do café apresentam uma taxa de crescimento dessa variável mais elevada que microrregiões de maior produtividade com o passar do tempo.

No que tange à Aede, em trabalhos para a economia agrícola brasileira, é possível citar, entre outros, Perobelli et al. (2007), que estudam a distribuição espacial da produtividade do setor agrícola entre as 558 microrregiões brasileiras, Souza e Perobelli (2008), que investigam a distribuição espacial da sojicultura para 
as 558 microrregiões brasileiras, e Souza e Perobelli (2007), que analisam a concentração espacial produtiva do setor cafeeiro, novamente entre as 558 microrregiões brasileiras.

Com relação à convergência espacial, é importante elencar a existência de trabalhos como o de Rey e Montoury (1999), que aplicam a análise da convergência da renda sob a ótica da econometria espacial para a economia norte-americana, e o de Le Gallo e Ertur (2003), que utilizam a mesma metodologia para análise da distribuição do PIB da economia europeia. No Brasil, Perobelli, Faria e Ferreira (2007) utilizam a mesma metodologia para investigar o processo de convergência da renda entre os municípios mineiros de 1975 a 2003. Lopes (2004) avalia o processo de convergência da produtividade da terra para as 11 principais culturas brasileiras entre os estados produtores. Mais recentemente, Almeida, Perobelli e Ferreira (2008) investigam o processo de $\beta$ convergência da produtividade agrícola no Brasil entre os diferentes estados, controlando os efeitos espaciais. Destarte, este trabalho constitui-se em uma aplicação desses métodos para a questão da produtividade média do café no estado de Minas Gerais.

De acordo com Lopes (2004), a convergência é um processo em que uma mesma variável (por exemplo, renda per capita ou produtividade da terra), apresenta diferentes valores entre países, regiões ou estados, mas essa diferença se reduz ao longo do tempo, indicando que a desigualdade ou discrepância entre os valores da variável analisada diminui ou é eliminada.

O trabalho seminal de Baumol (1986) examina a convergência da renda per capita, no período de 1870 a 1979, entre 16 países industrializados, utilizando-se da seguinte equação:

$$
\ln (Y / N)_{i, t}-\ln (Y / N)_{i, t-1}=\alpha+\beta \ln (Y / N)_{i, t-1}+\varepsilon_{i}
$$

em que $\ln (Y / N)$ é o logaritmo natural da renda per capita, $\varepsilon$ é o termo de erro bem comportado com média zero e variância constante, e i é o indexador para diversos países.

Segundo Baumol (1986), ocorre convergência quando o coeficiente $\beta$ da equação 1 for negativo e significativo, indicando que países com renda inicial maior crescerão a taxas menores que países com renda inicial menor, mostrando que, ao longo de tempo, os diversos países convergiriam para uma renda comum.

A exemplo de Baumol (1986), o presente estudo tem como um de seus objetivos averiguar a hipótese de convergência. Vale destacar que este trabalho irá adaptar a ideia apresentada por Baumol (1986) para entender o comportamento da produção de café em Minas Gerais. Ou seja, buscar-se-á verificar se microrregiões com baixa produtividade média de café estão crescendo de forma mais acentuada para essa variável que as microrregiões de elevada produtividade. 
Para fazer essa análise, o presente artigo faz uso da econometria espacial, pois, conforme Quah (1996), a maioria dos estudos sobre convergência utiliza dados regionais, mas grande parte deles trata as unidades regionais como localidades isoladas, ou seja, não considera a interação com seu entorno. Nesse mesmo sentido, de acordo com Rey e Montoury (1999), o desenvolvimento da econometria espacial e de ferramentas de Aede permitiu uma nova percepção da dinâmica geográfica dos padrões de crescimento no tempo.

Segundo Anselin (1999), a econometria espacial é um subcampo da econometria que lida com as complicações causadas pela interação espacial (autocorrelação espacial) e pela estrutura espacial (heterogeneidade espacial) em modelos de regressão com dados em corte cruzado ou em painel.

A autocorrelação espacial refere-se à dependência espacial causada pela interação dos distintos agentes no espaço. De forma geral, todo processo que se dá no espaço está sujeito à chamada Lei de Tobler, também conhecida como a Primeira Lei da Geografia, cujo enunciado pode ser estabelecido da seguinte forma: "tudo depende de todo o restante, porém o que está mais próximo depende mais do que aquilo que está mais distante". Essa lei destaca o papel da proximidade geográfica para o estabelecimento da interação espacial entre os fenômenos em estudo.

Por sua vez, a heterogeneidade espacial manifesta-se quando ocorre instabilidade estrutural no espaço, fazendo com que uma dada variável apresente diferentes respostas, dependendo da localidade ou da escala espacial. Tal heterogeneidade pode ser identificada na forma de coeficientes variáveis, de variância não constante, ou, ainda, de formas funcionais diferentes para determinados subconjuntos de dados. A consequência prática é a inadequação do ajuste de um mesmo modelo para todo o conjunto de dados.

É importante frisar que a agricultura é muito sensível aos efeitos espaciais. As culturas agrícolas se desenvolvem de maneira heterogênea através do espaço, independentemente do zoneamento geográfico de uma determinada localidade. Isso se deve a fatores como diferentes técnicas de produção, clima, topografia e condições de solo. Por outro lado, os efeitos de interdependência nas diferentes regiões produtoras manifestam-se de diversas maneiras, tais como: ordenação geográfica dos dados, difusão espacial de certos fenômenos que influenciam os vizinhos e processos de competição espacial no âmbito da expansão da fronteira agrícola ou na formação de cinturões agrícolas.

Além desta introdução, o trabalho está dividido em mais três seções. A seção 2 expõe de maneira detalhada a metodologia e o banco de dados utilizados, a seção 3 trata dos resultados da Aede e da econometria espacial e, por fim, a seção 4 trata das considerações finais. 


\section{Metodologia e Base de Dados}

A utilização de técnicas que captem de maneira explícita os efeitos da autocorrelação espacial entre distintas localidades mostram-se fundamentais em estudos com dados georeferenciados. A Aede e a econometria espacial, através do modelo de convergência espacial, são duas ferramentas que realizam essa função, como será explicado a seguir.

\subsection{Análise Exploratória de Dados Espaciais (Aede)}

A Aede está baseada em aspectos espaciais da base de dados, tratando diretamente de dependência espacial (associação espacial) e heterogeneidade espacial. Em outras palavras, a finalidade da Aede é caracterizar a distribuição espacial e os padrões de associação espacial (clusters espaciais), verificar a ocorrência de diferentes regimes espaciais ou outras formas de instabilidade espacial (não estacionariedade) e identificar observações atípicas (outliers) (PEROBELLI; FARIA; FERREIRA, 2007).

O primeiro passo para que a Aede e as técnicas de econometria espacial, como será visto adiante, sejam implementadas de maneira eficiente é a utilização de variáveis intensivas ou espacialmente densas, já que variáveis absolutas podem induzir a enganos na análise. A utilização do total de observações em números absolutos de um fenômeno não é aconselhável, tendo em vista que esses atributos podem estar correlacionados com variáveis de escala, o que geraria correlações espaciais espúrias (ANSELIN, 2005, 1988).

O segundo passo é a definição de uma matriz de pesos espaciais $W$, como forma de expressar o arranjo espacial das interações do fenômeno a ser estudado. Há na literatura um grande número de matrizes de pesos espaciais. É possível implementar uma Aede através de uma matriz de contiguidade binária, como a rainha (queen) ou a torre (rook). A matriz rainha considera como vizinhas localidades que possuem fronteira comum, além de nós (vértices). Já a matriz torre, por definição, é mais simples e considera como vizinhas somente localidades que possuem fronteira comum (ALMEIDA; PEROBELLI; FERREIRA, 2008).

Outra maneira de se expressar a estrutura de conectividade é por meio da distância geográfica. Uma alternativa nesses casos é utilizar uma matriz de pesos espaciais $W$, baseada nos $k$ vizinhos mais próximos, $w_{i j}(k)$, que definiria a força da interação entre as regiões (PEROBELLI et al., 2007).

A matriz de pesos espaciais $W$ utilizada neste trabalho baseia-se na ideia de $k$ vizinhos mais próximos. Ela foi calculada utilizando o conceito de grande círculo entre os centros das regióes, ou seja, a menor distância entre dois pontos quais- 
quer na superfície de uma esfera. A especificação da matriz $W$ com base nos $k$ vizinhos mais próximos é a seguinte:

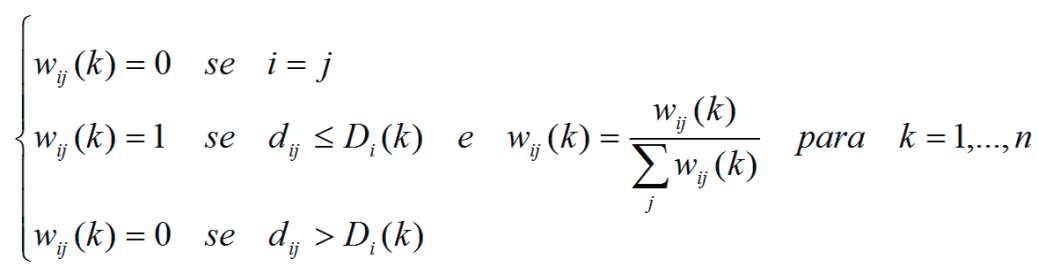

em que $d_{i j}$ é a distância, medida pelo grande círculo, entre os centros das regiões $i$ e j; $D_{i}(k)$ é o valor crítico de corte, que define a distância máxima para considerar regiões vizinhas à região i. Em outras palavras, distâncias acima desse ponto não serão levadas em consideração, ou seja, as regiões não serão tomadas como vizinhas (SOUZA; PEROBELLI, 2008).

Durante a elaboração deste trabalho, foram testadas as matrizes de contiguidade rainha e torre, bem como matrizes de distância geográfica para $k=5, k=10$, $k=15$ e $k=20$ vizinhos mais próximos. A matriz $k=10$ vizinhos é a que será considerada na análise. Isso se justifica porque essa matriz forneceu os resultados espacialmente mais robustos. Todavia, é importante frisar que todas as demais matrizes testadas revelaram-se estatisticamente significativas, corroborando a existência de autocorrelação espacial global. ${ }^{1}$

\subsubsection{Autocorrelação Espacial Global}

Uma forma de calcular a autocorrelação espacial global é por meio do I de Moran. Essa é uma estatística que fornece de maneira formal o grau de associação linear entre os vetores de valores observados em um tempo t(z) e a média ponderada dos valores dos seus vizinhos, ou as defasagens espaciais $\left(W z_{t}\right)$, como são mais conhecidos na literatura. Valores do I de Moran maiores ou menores do que o valor esperado $E(I)=-1 /(n-1)$ revelam autocorrelação espacial positiva ou negativa, respectivamente (ALMEIDA; HADDAD; HEWINGS, 2005; PEROBELLI et al. 2007).

Os valores dessa estatística variam entre -1 e +1 , em que -1 representa um coeficiente de correlação linear perfeitamente negativo e +1 representa um coeficiente de correlação linear perfeitamente positivo (ANSELIN, 1995). Para Cliff e Ord (1981), essa estatística pode ser representada através da seguinte notação matricial:

$\overline{1}$ Os resultados para as matrizes rainha e torre, bem como para as matrizes $k=5, k=15$ e $k=20$ podem ser obtidos diretamente com os autores.

TEIXEIRA, R. F. A. P.; BERTELLA, M. A. Distribuição Espaço-Temporal da Produtividade... 


$$
I_{t}=\left(\frac{n}{S_{o}}\right)\left(\frac{z_{t}^{\prime} W z_{t}}{z_{t}^{\prime} z_{t}}\right) t=1, \ldots n
$$

em que $z_{t}$ é o vetor de $n$ observações para o ano $t$ na forma de desvio em relação à média; Wé a matriz de pesos espaciais que, conforme Pimentel e Haddad (2004), pode ser definida como uma matriz quadrada em que cada célula $w_{i j}$ indica relação existente entre a região $i$ e j em um sistema de $n$ regiões, como indicado na equação 2; e o termo $S_{0}$ é um escalar igual à soma de todos os elementos de W.

A matriz de pesos espaciais quando normalizada na linha, isto é, quando a soma dos elementos da linha é igual a um, faz com que a expressão 3 assuma a seguinte forma:

$$
I_{t}=\left(\frac{z_{t}^{\prime} W z_{t}}{z_{t}^{\prime} z_{t}}\right) t=1, \ldots n
$$

Anselin (1995) considera que o I de Moran, por ser uma medida de associação espacial global, não é capaz de revelar padrões de associação espacial local. Para isso, existe a autocorrelação espacial local.

\subsubsection{Autocorrelação Espacial Local}

A estatística global do I de Moran pode esconder padrões locais de autocorrelação espacial. É possível ocorrer três situações distintas. A primeira envolve a indicação de um I de Moran global insignificante, do ponto de vista estatístico. Porém, podem existir indicações de autocorrelação espacial local significante, positiva ou negativa. A segunda situação implica uma indicação positiva do I de Moran global, que oculta autocorrelação espacial local negativa e significante do ponto de vista estatístico. A terceira situação denota que a evidência de uma autocorrelação espacial global negativa pode acomodar indícios de autocorrelação espacial local positiva para certos grupos de dados. Em decorrência desses efeitos, é importante avaliar o padrão local da autocorrelação espacial, de modo a se obter um maior detalhamento do fenômeno em estudo (PEROBELLI et al., 2007).

A fim de se observar a existência de clusters espaciais locais de valores altos ou baixos e quais regiões mais contribuem para a existência de autocorrelação espacial, deve-se implementar medidas de autocorrelação espacial local, quais sejam: o diagrama de dispersão de Moran (Moran scatterplot) e os indicadores locais de associação espacial (Lisa). ${ }^{2}$

$2 \quad$ No acrônimo em inglês, Local Indicators of Spatial Association. 


\subsubsection{Diagrama de Dispersão de Moran}

O diagrama de dispersão de Moran é uma das formas de interpretar a estatística I de Moran. Trata-se de uma representação do coeficiente de regressão linear, mediante um gráfico de duas variáveis $z$ e Wz, na qual o coeficiente da inclinação da curva de regressão é dado pela estatística I de Moran. A inclinação da curva é obtida pela regressão de $\mathrm{Wz}$ contra $z$, e essa inclinação fornece o grau de ajustamento (ANSELIN, 1998, 1995).

O diagrama de dispersão de Moran (ver Figura 1) é dividido em quatro quadrantes que correspondem a quatro padrões de associação espacial local entre determinadas regiões e seus respectivos vizinhos.

Figura 1 - Diagrama de dispersão de Moran

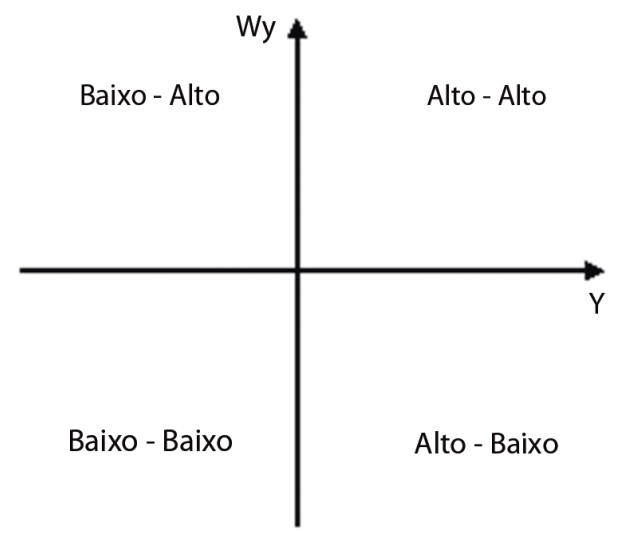

Fonte: Elaboração própria.

O primeiro quadrante, conhecido como alto-alto (AA), localiza-se na parte superior direita e representa regiões que possuem valores acima da média para uma determinada variável cercada por regiões que também possuam valores acima da média para essa variável.

O segundo quadrante, denominado de baixo-alto (BA), localiza-se na parte superior esquerda e é constituído por regiões que apresentam baixos valores para a variável de interesse, rodeadas por regiões que possuem elevado valor para essa mesma variável.

O terceiro quadrante, classificado como baixo-baixo (BB), está localizado na parte inferior esquerda e indica regiões que possuem baixos valores para a variável em análise, cercadas por regiões de também baixo valor para a mesma variável.

O quarto e último quadrante, conhecido como alto-baixo (AB), encontra-se localizado na parte inferior direita e revela regiões que possuem valores acima 
da média para a variável de interesse, ladeadas por regiões que possuem valores abaixo da média para a mesma variável.

Como destacam Perobelli et al. (2007), as regiões que apresentam padrões de associação espacial positiva AA e BB formam clusters de valores similares, ao passo que regiões de padrões $\mathrm{BA}$ e $\mathrm{AB}$ apresentam associação espacial negativa.

\subsubsection{Indicadores Locais de Associação Espacial (Lisa)}

Os indicadores Lisa indicam o grau de autocorrelação espacial local. Conforme enfatiza Anselin (1999, 1995), para que isso ocorra é necessário que essa estatística satisfaça dois critérios: a) esses indicadores devem possuir para cada observação uma indicação de clusters espaciais significantes de valores similares ao redor de cada observação; e b) o somatório dos indicadores Lisa, em todas as regiões, deve ser proporcional ao indicador de autocorrelação espacial global. Dessa maneira, os indicadores Lisa podem ser representados por intermédio da seguinte equação:

$$
I_{i}=\frac{\left(y_{i}-\bar{y}\right) \sum_{j} w_{i j}\left(y_{j}-\bar{y}\right)}{\sum_{i}\left(y_{i}-\bar{y}\right)^{2} / n}
$$

na qual $n$ é o número de regiões, $y_{i}$ é a variável de interesse, $\bar{y}$ é a média dessa variável, $y_{j}$ é a variável de interesse nas regiões vizinhas a $i$ e $w_{i j}$ é o elemento da matriz de pesos espaciais.

De acordo com o mesmo autor, a estatística Lisa é usada para testar a hipótese nula, ou seja, a ausência de associação espacial local. Assim, deve-se fazer uso de uma aleatorização condicional, que permita determinar pseudoníveis de significância.

Para a obtenção de uma distribuição empírica das estatísticas de teste, deve-se observar se o valor da variável de interesse está dentro ou fora da região crítica definida. Dessa maneira, se o valor calculado for superior em magnitude à esperança matemática do I de Moran, seus resultados serão estatisticamente significativos.

\subsection{Análise de Convergência Espacial}

Ao se definir um modelo de convergência que possa captar de maneira adequada questões inerentes ao processo de autocorrelação espacial, os efeitos espaciais devem ser explicitamente considerados em sua forma funcional. Modelos 
de convergência tradicionais, que se utilizam de regressão linear, não levam em conta os efeitos espaciais da base de dados. Desse modo, a estimação desse tipo de modelo por mínimos quadrados ordinários (MQO) forneceria estimativas inconsistentes e/ou ineficientes (ANSELIN, 1995, 1988; ANSELIN; BERA, 1998).

Autores como Florax, Folmer e Rey (2003) e Anselin (2005) sugerem o seguinte procedimento para a especificação de um modelo de convergência que leve em conta a presença de autocorrelação espacial:

a) estimar o modelo clássico de regressão linear por MQO;

b) testar a hipótese de ausência de autocorrelação espacial devido a uma defasagem ou a um erro através do multiplicador de Lagrange para a defasagem espacial $\left(M L_{\rho}\right)$ e do multiplicador de Lagrange para o erro espacial $\left(M L_{\lambda}\right)$;

c) caso ambos os testes sejam não significativos, deve-se utilizar o modelo clássico. Caso contrário, segue-se o próximo passo;

d) se ambos os testes forem significativos, deve-se verificar as versões robustas de ambos, ou seja, o multiplicador de Lagrange robusto para a defasagem espacial $\left(M L_{\rho}\right)$ e o multiplicador de Lagrange robusto para o erro espacial $\left(M L_{\lambda}\right)$. Caso $M L_{\rho}>M L_{\lambda}$, identifica-se o modelo de defasagem espacial como o mais apropriado. Caso contrário, $M L_{\rho}<M L_{\lambda}$, adota-se o modelo de erro espacial.

A estimação do modelo de $\beta$ convergência absoluta por MQO busca somente identificar qual a melhor maneira de se estimar a equação abaixo:

$$
\ln \left(\text { Pmec }_{t} / \text { Pmec }_{t-n}\right)=\alpha+\beta \ln \left(\text { Pmec }_{t-n}\right)+u_{i}
$$

em que $\ln \left(\right.$ Pmec $_{t} /$ Pmec $\left._{t-n}\right)$ é o logaritmo natural da razão entre produtividade média do café no período final e inicial; $\beta \ln \left(\mathrm{Pmec}_{t-n}\right)$ é o logaritmo natural da produtividade média do café no período inicial; e $u_{i}$ é o termo de erro bem comportado.

A seguir, conforme Rey e Montoury (1999), são expostos os três modelos de convergência mais utilizados pela literatura para analisar o processo de dependência espacial dos dados, a saber: os modelos de erro espacial, de defasagem espacial e o regressivo cruzado. Cada um deles fornece um tipo diferente de arranjo entre as unidades regionais para explicar os efeitos que podem surgir da interação espacial. 


\subsubsection{Modelo de Erro Espacial}

A primeira modificação que a equação 6 pode sofrer refere-se ao seu termo de erro $u_{i}$, que pode seguir um processo autorregressivo, como mostrado na equação 7:

$$
u_{i}=\lambda W u_{i}+\varepsilon_{i}
$$

em que $\lambda$ representa o coeficiente escalar do erro espacial, e o termo de erro $\varepsilon_{i}$ possui distribuição normal com média zero e variância constante. Ao se substituir a equação 7 na equação 6 , tem-se a forma convencional do modelo de regressão do erro espacial:

$$
\ln \left(\text { Pmc }_{t} / \text { Pmec }_{t-n}\right)=\alpha+\beta \ln \left(\text { Pmec }_{t-n}\right)+(I-\lambda W) \varepsilon_{i}
$$

Segundo Rey e Montoury (1999), quando $\lambda$ assume valor nulo, não há autocorrelação espacial do erro. Contudo, quando $\lambda \neq 0$, um choque ocorrido em uma unidade geográfica se espalha não somente para seus vizinhos mais próximos, mas para todas as outras unidades. Isto é, a dependência espacial pode ser decorrente de efeitos não modelados que não foram aleatoriamente distribuídos através do espaço.

A utilização de $\mathrm{MQO}$ na presença de erros não esféricos geraria estimativas ineficientes, apesar de justas. Nesse aspecto, o modelo de erro espacial deve ser estimado pelo método da máxima verossimilhança (MV) ou pelo método generalizado dos momentos (MGM) de Kelejian e Prucha (1999).

\subsubsection{Modelo de Defasagem Espacial}

Esse modelo sugere que a autocorrelação espacial é fruto da interação atual entre as regiões. Nessa nova equação de $\beta$ convergência, é introduzida como variável independente uma defasagem espacial da variável dependente. O modelo é dado pela seguinte formulação:

$$
\ln \left(\text { Pmec }_{t} / \text { Pmec }_{t-n}\right)=\alpha+\beta \ln \left(\text { Pmec }_{t-n}\right)+\rho W \ln \left(\text { Pmec }_{t} / \text { Pmec }_{t-n}\right)+\varepsilon_{i}
$$

na qual $\rho$ é o coeficiente da defasagem espacial, isto é, um escalar em que $\rho>0$ $(\rho<0)$ indica ocorrência de autocorrelação espacial positiva (negativa). O novo 
elemento $W \ln \left(\mathrm{Pmec}_{t} /\right.$ Pmec $\left._{t-n}\right)$ pode ser entendido como uma média dos valores da taxa de crescimento das unidades espaciais vizinhas.

De acordo com Anselin (1988), ao não se considerar explicitamente a defasagem espacial nesse modelo, ocorreria um problema da mesma magnitude da omissão de variável relevante, isto é, os coeficientes estimados por MQO das variáveis explicativas seriam tendenciosos. Para evitar tal problema, métodos de estimação alternativos, como o uso de variáveis instrumentais (VI), ou o método MV, são recomendados.

\subsubsection{Modelo Regressivo Cruzado Espacial}

Esse modelo trata dos efeitos de transbordamento espacial. No contexto da convergência, o efeito de transbordamento é representado pela defasagem espacial da variável independente. Formalmente, o modelo é dado pela seguinte expressão:

$$
\ln \left(\text { Pmec }_{t} / \text { Pmec }_{t-n}\right)=\alpha+\beta \ln \left(\text { Pmec }_{t-n}\right)+\tau W \ln \left(P m e c_{t-n}\right)+\varepsilon_{i}
$$

na qual $\tau$ é o coeficiente de transbordamento, e o novo elemento $W \ln \left(\right.$ Pmec $\left._{t-n}\right)$ denota a defasagem espacial da variável independente, no período inicial, nas regiões vizinhas. Como a defasagem espacial da variável independente é uma variável exógena, pode-se estimar esse modelo por MQO (REY; MONTOURY, 1999).

\subsubsection{Dados}

Este estudo fez uso dos softwares Geodata Analysis (GeoDa) e Spacestat 1.91, além da base de dados da Pesquisa Agrícola Municipal (PAM), gerada por meio do Sistema IBGE de Recuperação Automática (Sidra), do qual se extraíram informações referentes à produtividade média de café (quilogramas por hectare), de maneira a respeitar a literatura sobre o assunto, que enfatiza a utilização de variáveis intensivas.

Dessa maneira, pretendeu-se mensurar, de modo mais coerente, a existência de autocorrelação espacial global e local, bem como a ocorrência de $\beta$ convergência absoluta na produtividade média de café entre as 66 microrregiões mineiras durante o período 1997-2006. É importante frisar que esse período foi o escolhido por apresentar uma enorme expansão da produtividade cafeeira no estado, que se incrementou de 10 sacas por hectare (ha) para aproximadamente 20 sacas, isto 
é, um desempenho excepcional para um período de dez anos (BRASIL, 2009). Além do mais, vários trabalhos citados anteriormente, como Perobelli et al. (2007), Souza e Perobelli $(2007,2008)$, têm apresentado análises para diferentes culturas agrícolas, através de uma abordagem com microrregiões.

\section{Análise dos Resultados}

Antes de se analisar os padrões de autocorrelação espacial, é importante observar o desempenho da produtividade média do café entre as microrregiões mineiras sem a adoção dos efeitos espaciais. Destarte, foram construídos três mapas de quartis, um para o ano de 1997, um para o ano de 2006 e um referente à taxa de crescimento do período 1997-2006, conforme a Figura 2.

Figura 2 - Produtividade média do café (Quartis)

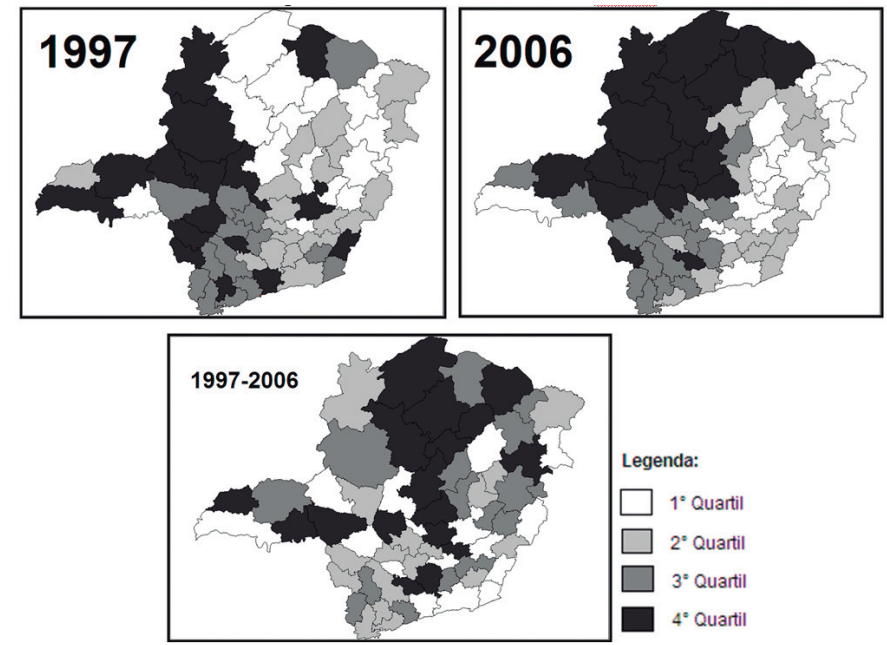

Fonte: Elaboração própria com base no programa GeoDa.

Como pode ser visualizada, a produtividade média do café em Minas Gerais teve sua configuração alterada em um período de dez anos. No ano de 1997, as microrregiões de elevada produtividade (quarto quartil) concentravam-se, mormente, na parte oeste do estado. Já no ano de 2006, esse arranjo se alterou, com as microrregiões de alta produtividade localizando-se de maneira destacada nas porções norte e centro-oeste.

Essa dinâmica pode ser mais bem compreendida através da taxa de crescimento da produtividade média do café durante o período 1997-2006. Ao longo desses dez anos, as microrregiões concentradas na porção centro-norte do estado foram as que apresentaram maiores taxas de crescimento na produtividade dessa 
cultura, juntamente com algumas microrregiões da porção oeste, com esse crescimento variando entre $36,3 \%$ e $507 \%$ (quarto quartil). ${ }^{3}$

A mudança sofrida na produtividade do setor cafeeiro entre as microrregiões mineiras indica um reordenamento dessa variável ao longo do tempo. Entretanto, para uma análise mais completa, é necessário reconhecer a influência de uma localidade sobre sua vizinhança e vice-versa. Nesse sentido, a estatística I de Moran fornece, de maneira formal, o grau de associação espacial entre as distintas microrregiões.

\subsection{Estatística I de Moran}

O I de Moran é capaz de revelar a presença de autocorrelação espacial entre distintas localidades. A Tabela 1 refere-se ao I de Moran global, sua média, seu desvio padrão e o z-value para a produtividade média do café durante os anos de 1997, 2006 e para a taxa de crescimento do período (1997-2006).

Tabela 1 - I de Moran: teste de autocorrelação espacial

\begin{tabular}{lllll}
\hline Período & I de Moran & Média & Desvio padrão & z-value \\
\hline 1997 & 0,2469 & $-0,015$ & 0,046556 & 5,633851 \\
$1997-2006$ & 0,1746 & $-0,015$ & 0,046556 & 7,240423 \\
2006 & 0,3217 & $-0,015$ & 0,046556 & 4,080551 \\
\hline
\end{tabular}

Fonte: Elaboração própria a partir do programa SpaceStat.

Em todos os períodos, o I de Moran apresentou valores positivos e significativos, indicando que existe autocorrelação espacial global positiva em todas as análises. Em outras palavras, microrregiões com elevada produtividade média do café são circundadas por microrregiões com a mesma característica, ou, alternativamente, microrregiões com baixa produtividade média do café são vizinhas de outras microrregiões que também ostentam baixa produtividade média para a mesma variável.

Outro fator importante pode ser constatado pela evolução espaço-temporal dessa estatística, que se elevou de 0,2469, em 1997, para 0,3217, em 2006, indicando que a dependência espacial entre as microrregiões mineiras aumentou. Por sua vez, para a taxa de crescimento da produtividade média do café no período

3 As microrregiões do primeiro quartil indicaram somente decréscimo da atividade cafeeira, com suas taxas situando-se entre $-21,5 \%$ e $-72,4 \%$. Já as microrregiões presentes no segundo quartil revelaram taxas entre $-20,5 \%$ e $0,2 \%$, isto é, essas localidades podem ser caracterizadas pela estagnação ou pelo decréscimo da cafeicultura. Por fim, nesse mesmo período, as microrregiões do terceiro quartil obtiveram taxas de crescimento, também positivas, entre 3,1\% e 35,5\%.

TEIXEIRA, R. F. A. P.; BERTELLA, M. A. Distribuição Espaço-Temporal da Produtividade... 
(1997-2006), o I de Moran apresentou um valor de 0,1746, sugerindo que o crescimento dessa cultura em Minas Gerais não segue um processo aleatório.

\subsection{Diagrama de Dispersão de Moran}

O diagrama de dispersão de Moran é uma ferramenta interessante porque revela padrões locais de associação espacial e não apenas a tendência geral de agrupamento dos dados, como o I de Moran. A Figura 3 representa os diagramas de dispersão de Moran para a produtividade média do café nas microrregiões mineiras nos anos de 1997 e 2006 e a taxa de crescimento da produtividade média (1997-2006), respectivamente.

Figura 3 - Diagrama de dispersão de Moran da produtividade média de café nas microrregiões mineiras
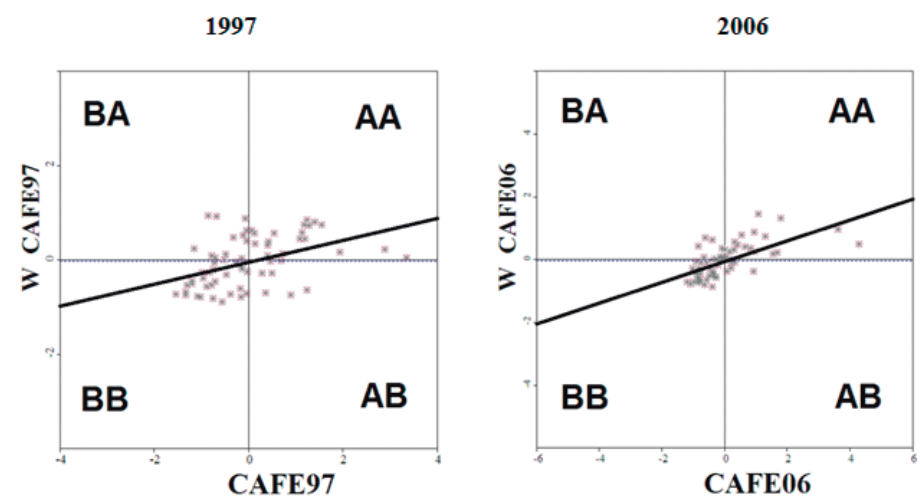

1997-2006

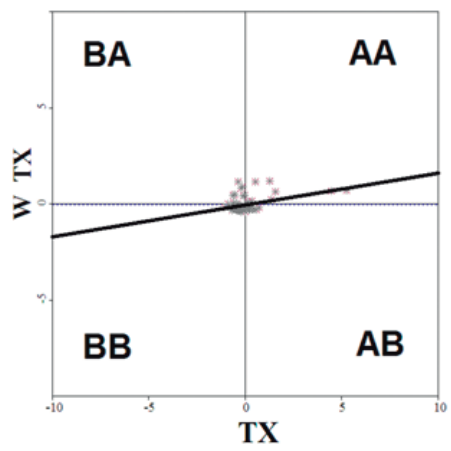

Fonte: Elaboração própria a partir do programa GeoDa. 
O primeiro diagrama de dispersão de Moran refere-se ao ano de 1997 e mostra que $69,7 \%$ das microrregiões mineiras possuíam associação espacial positiva, ou seja, quase $70 \%$ das microrregiões em Minas Gerais apresentavam ou o padrão AA, ou o padrão BB durante esse ano. Por sua vez, essa mesma estatística revelou que, no ano de 2006, esse valor elevou-se para 80,3\%. Com relação à taxa de crescimento da produtividade média do café no período (1997-2006), pode-se constatar que 68,2\% das microrregiões contavam com associação espacial positiva.

Apesar do diagrama de dispersão de Moran fornecer os padrões locais de associação espacial, ele não é capaz de indicar a existência de clusters significativos na produtividade média de café. Para tanto, faz-se uso da estatística Lisa.

\subsection{Indicadores Locais de Associação Espacial (Lisa)}

Os indicadores Lisa para a produtividade média do café nas microrregiões mineiras durante os anos de 1997 e 2006 e para o período 1997-2006 estão presentes nos mapas de clusters, que podem ser visualizados na Figura 4.

Figura 4 - Mapas de clusters

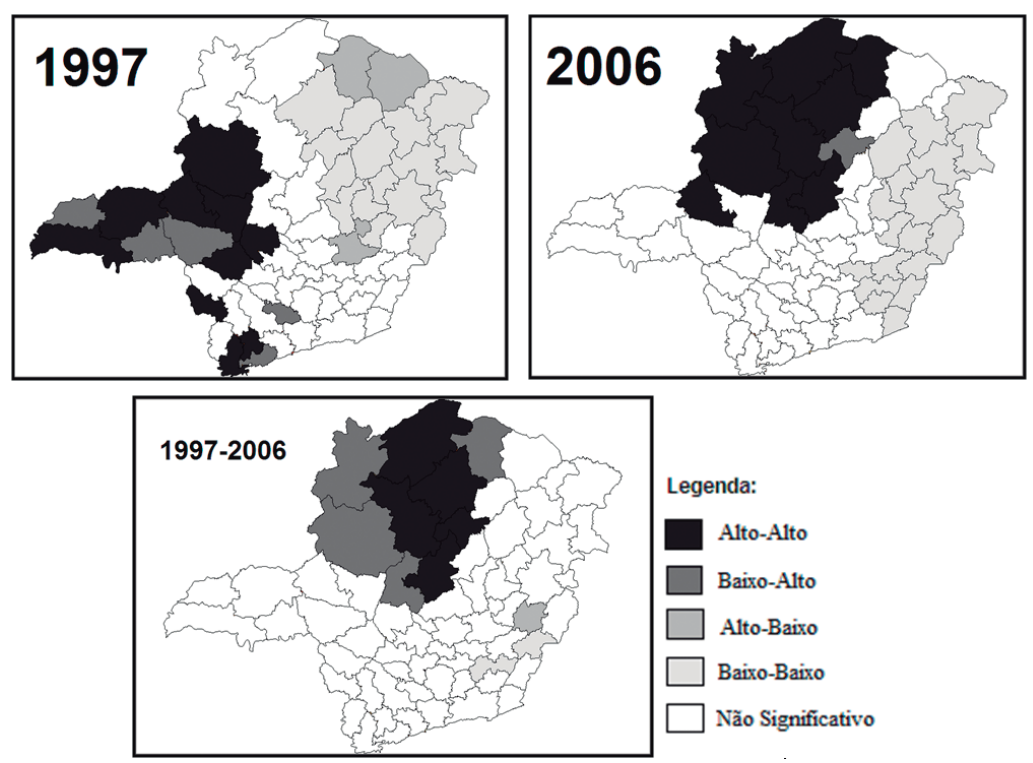

Fonte: Elaboração própria a partir do programa GeoDa. 
O mapa de clusters para a produtividade média do café durante o ano de 1997 revelou os quatro tipos de associação espacial possíveis, ou seja, os padrões AA, BB, AB e BA. O padrão AA localiza-se nas porções oeste e sul do estado, sendo representado pelas microrregiões de Bom Despacho, Piumhi, Paracatu, Frutal, Patos de Minas, Patrocínio, Uberlândia, Pouso Alegre, Santa Rita do Sapucaí e São Sebastião do Paraíso, que possuem uma agricultura moderna, respaldada em tecnologia avançada.

Por sua vez, o cluster BB localiza-se, mormente, nas regiões central e nordeste do estado, sendo representado pelas microrregiões de Almenara, Araçuaí, Capelinha, Diamantina, Pedra Azul, Bocaiúva, Montes Claros, Grão Mogol, Aimorés, Guanhães, Governador Valadares, Ipatinga, Mantena, Peçanha, Nanuque, Teófilo Otoni e Conceição do Mato Dentro. Isto é, esse cluster de baixa produtividade ou localiza-se em microrregiões de maior industrialização, como é o caso de Governador Valadares e Ipatinga, ou em microrregiões reconhecidamente pobres, por exemplo, Almenara e Capelinha.

De maneira distinta aos padrões $\mathrm{AA}$ e $\mathrm{BB}$, os padrões $\mathrm{BA}$ e $\mathrm{AB}$ revelam a chamada autocorrelação espacial negativa. $O$ padrão $A B$ é representado pelas microrregiões de Itabira, Janaúba e Salinas. Ou seja, são localidades que possuem alta produtividade média de café e que são vizinhas de regiões com baixa produtividade para essa mesma variável. As microrregiões de Janaúba e Salinas, mesmo fazendo parte do norte de Minas, possuem elevada produtividade cafeeira, principalmente pelo uso intensivo da mão de obra. Por fim, o padrão BA encontra-se nas porções oeste e sul do estado, quase sempre vizinhas às regiões de alta produtividade da cafeicultura. Esse padrão foi localizado nas microrregiões de Araxá, Ituiutaba, Uberaba, Lavras e Itajubá.

Já o mapa de clusters, para a produtividade média do café durante o ano de 2006, apresenta configuração distinta se comparado ao ano de 1997. Esse mapa revela, basicamente, dois grandes clusters: um de alta produtividade na porção noroeste do estado e outro de baixa produtividade nas regiões nordeste e sudeste de Minas Gerais.

O primeiro cluster AA é composto pelas microrregiões de Curvelo, Três Marias, Januária, Montes Claros, Pirapora, Paracatu, Unaí e Patrocínio. Por sua vez, o padrão BB é composto pelas microrregiões de Aimorés, Caratinga, Governador Valadares, Guanhães, Ipatinga, Mantena, Peçanha, Almenara, Araçuaí, Capelinha, Cataguases, Manhuaçu, Muriaé, Ponte Nova, Viçosa, Nanuque e Teófilo Otoni.

Comparando o cluster AA de 2006 com o de 1997, observa-se uma alteração significativa, pois somente as microrregiões de Paracatu e Patrocínio permaneceram em ambas as análises. Por outro lado, ao se defrontar o cluster BB de 2006 com o de 1997, não foi encontrada nenhuma mudança muito significativa. 
O destaque fica por conta da entrada de algumas microrregiões da Zona da Mata Mineira nesse cluster de baixa produtividade.

Por fim, cabe destacar o padrão BA presente na microrregião de Bocaiúva. Essa localidade mostra-se como uma verdadeira ponte de ligação entre o padrão $\mathrm{AA}$ e o padrão $\mathrm{BB}$. O padrão $\mathrm{BA}$ indica que essa microrregião possuía uma baixa produtividade média de café em 2006, mas tinha que como vizinhas localidades que desfrutavam de elevada produtividade média para a mesma variável.

A análise referente ao período 1997-2006 revela a tendência espaço-temporal da taxa de crescimento da produtividade média do café. Pode-se visualizar que microrregiões ao norte do estado apresentaram os padrões AA e BA, enquanto que, na região sudeste, duas microrregiões revelaram o padrão BB e uma o padrão $\mathrm{AB}$.

O comportamento da produtividade média ao longo do tempo e do espaço, como mostrado na Figura 4, indica um rearranjo da estrutura produtiva do café em Minas Gerias. Em outras palavras, parece que está ocorrendo um processo de convergência dessa variável entre as distintas microrregiões, indicando que a produtividade média do setor cafeeiro tende a se tornar mais homogênea em todo o estado. No entanto, os mapas de clusters fornecem apenas indicações de tendência espacial, sem conseguirem indicar de maneira formal a ocorrência do processo de convergência. Para tanto, modelos de $\beta$ convergência devem ser testados através da econometria espacial.

\subsection{Convergência Espacial}

A regressão da equação 6 permite obter a estimativa de $\beta$, que estuda a hipótese de que as microrregiões com baixa produtividade média do café tendem a elevar essa variável mais rapidamente que microrregiões com alta produtividade média.

O modelo de $\beta$ convergência clássico estimado por MQO está representado resumidamente na Tabela 2.

Tabela 2 - Resultados iniciais

\begin{tabular}{lc}
\hline \multicolumn{2}{c}{ Modelo clássico - MQO } \\
\hline Coeficientes & $\mathbf{1 9 9 7 - 2 0 0 6}$ \\
\hline$\alpha$ & $-0,7047$ \\
& $(0,0000)$ \\
$\beta$ & $-0,4098$ \\
& $(0,0000)$ \\
\hline
\end{tabular}

continua... 
conclusão.

\section{Estatísticas}

AIC

67,6657

SC

72,045

LIK

$-31,8329$

I de Moran

0,1206

$(0,0009)$

ML - erro

5,4514

$(0,0195)$

ML - defasagem

0,2845

$(0,5938)$

MLR - erro

9,7262

$(0,0195)$

MLR - defasagem

4,5593

$(0,0327)$

Teste Breusch-Pagan

7,6453

$(0,0057)$

Teste Jarque-Bera

Fonte: Elaboração própria a partir do programa SpaceStat.

Nota: As probabilidades estão entre parênteses.

Estimou-se primeiramente o modelo de regressão linear clássico por MQO, o qual, por meio do conjunto dos testes, verifica a hipótese de autocorrelação espacial na modelagem. As estatísticas de teste do multiplicador de Lagrange e do multiplicador de Lagrange robusto são as que permitem inferir não somente a existência de autocorrelação espacial, como também o melhor modelo econométrico espacial a ser utilizado, caso realmente exista a autocorrelação espacial (ALMEIDA; PEROBELLI; FERREIRA, 2008).

A primeira medida a ser tomada, após processar o modelo de $\beta$ convergência absoluta por MQO, é analisar o sinal do coeficiente $\beta$, que indicará ou não a existência de convergência. Como pode ser visualizado na Tabela 2, o modelo de regressão linear clássico revela a ocorrência de convergência, já que o coeficiente $\beta$ possui sinal negativo e também é estatisticamente significativo.

O segundo passo consiste em averiguar as estatísticas de teste do multiplicador de Lagrange. Somente o multiplicador de Lagrange do erro $\left(M L_{\lambda}\right)$ mostrou-se estatisticamente significativo, sugerindo que o modelo de erro espacial é o mais indicado para explicar o processo de convergência absoluta que ocorre na pro- 
dutividade média do café entre as microrregiões mineiras. Nesse caso, conforme indicado por Florax, Folmer e Rey (2003) e Anselin (1995), a análise do multiplicador de Lagrange robusto não se mostra necessária.

Entretanto, como forma de tornar o estudo mais interessante, foram processados todos os modelos propostos para que o leitor possa compará-los e, assim, averiguar a robustez do modelo de erro espacial.

É importante também sublinhar que o modelo estimado por MQO possui os erros distribuídos normalmente, como mostra o teste de Jarque-Bera. No entanto, a estimação sofre com o problema da heterocedasticidade, ou seja, variância não constante no termo de erro. O teste de Breusch-Pagan confirma essa hipótese.

Dessa forma, como sugere a literatura, os modelos de erro-espacial e defasagem-espacial podem ser estimados pelo método MV, pois possuem os erros distribuídos normalmente (ANSELIN, 1999). Caso a hipótese de normalidade nos erros não vigorasse, o MGM de Kelejian e Prucha (1999) seria o mais indicado. Por outro lado, o problema da ausência de homocedasticidade foi corrigido de forma a acomodar a heterocedasticidade em forma de grupos (groupwise heteroskedasticity), conforme Almeida, Perobelli e Ferreira (2008).

Como pode ser observado na Tabela 3, todos os modelos corroboram a hipótese do processo de convergência espacial através do sinal negativo do coeficiente $\beta$, revelando a robustez da análise. Além disso, o modelo de erro espacial, como esperado, mostrou-se o melhor dos modelos, mormente por apresentar os menores valores para os critérios de informação AIC (Akaike) e SC (Schwarz) e o maior valor para o critério LIK (valor da função log-verossimilhança). Outro ponto relevante que fornece respaldo ao modelo de erro espacial refere-se ao fato de o modelo de defasagem espacial possuir um coeficiente $\rho$ não estatisticamente significativo. Por sua vez, o modelo regressivo cruzado mostrou-se melhor que o modelo de defasagem espacial, mas pior que o modelo de erro espacial para explicar o processo de $\beta$ convergência na produtividade média do café entre as microrregiões mineiras, com um coeficiente $\tau$ mostrando-se estatisticamente significativo em nível de $5 \%$.

Tabela 3 - Modelos espaciais

\begin{tabular}{l|lll}
\hline \multirow{2}{*}{ Coeficientes } & \multicolumn{3}{|c}{ Modelos } \\
\cline { 2 - 4 } & Erro espacial & Defasagem espacial & Regressivo cruzado \\
\hline$\alpha$ & $-1,0058$ & $-0,6786$ & $-0,4358$ \\
& $(0,0000)$ & $(0,0000)$ & $(0,0185)$ \\
$\beta$ & $-0,5329$ & $-0,3921$ & $-0,5799$ \\
& $(0,0000)$ & $(0,0000)$ & $(0,0000)$ \\
\hline
\end{tabular}

continua... 


\begin{tabular}{l|lll} 
conclusão. & \multicolumn{3}{c}{ Modelos } \\
\hline$\lambda$ & 0,6314 & Defasagem espacial & Regressivo cruzado \\
\cline { 2 - 4 } & $(0,0000)$ & & \\
$\rho$ & & 0,1363 & \\
& & $(0,5264)$ & 0,3302 \\
$\tau$ & & & $(0,0344)$ \\
\hline Estatisticas & & & 64,9413 \\
\hline AIC & 58,5314 & 69,3483 & 71,5103 \\
SC & 62,9108 & 75,9173 & $-29,4706$ \\
LIK & $-27,2657$ & $-31,6742$ & 0,2801 \\
Teste & 0,2677 & 1,4478 & \\
Breusch- & & & $(0,5966)$ \\
Pagan & & $(0,2289)$ & \\
& $(0,6048)$ & & \\
\hline
\end{tabular}

Fonte: Elaboração própria a partir do programa SpaceStat.

Nota: As probabilidades estão entre parênteses.

Outra análise complementar e bastante interessante ao processo de $\beta$ convergência é o cálculo da taxa de convergência. De acordo com Rey e Montoury (1999), a taxa de convergência pode ser obtida através da expressão $\theta=-\ln (\beta+1) / k$, em que k é o número de anos no período de análise. A Tabela 4 mostra a taxa de convergência calculada para o período 1997-2006.

Tabela 4 - Taxa de convergência para o período 1997-2006

\begin{tabular}{c|c}
\hline 1997-2006 & Taxa de convergência $(\theta)$ \\
\hline$\theta$ & 0,0761 \\
\hline
\end{tabular}

Fonte: Elaboração própria.

Intuitivamente, essa taxa revela a velocidade com que ocorre o processo de convergência entre as distintas regiões ao longo do tempo (BARRO; SALA-I-MARTIN, 1992, 1991). Ou seja, uma taxa de convergência $\theta$ de 0,0761 indica que as disparidades regionais na produtividade média do café, no estado de Minas Gerais, estão se reduzindo a uma taxa de 7,61\% ao ano. Uma taxa de convergência de tamanha magnitude sugere que o processo de convergência está ocorrendo de maneira bastante rápida entre as microrregiões mineiras, indicando que localida- 
des, com baixa produtividade média do café no período inicial (1997), apresentam essa variável crescendo a taxas mais aceleradas que regiões com elevada produtividade média do café no período inicial.

\section{Considerações Finais}

Ao se analisar fenômenos que compreendam heterogeneidade espacial e dependência espacial, a econometria espacial procura incorporar interações e características estruturais que podem ser instáveis no espaço. A agricultura é um exemplo típico de fenômeno sensível aos efeitos espaciais, pois o espaço em que as culturas são desenvolvidas depende de diferentes condições de clima, solo e topografia, bem como da ordenação dos dados entre as diferentes regiões produtoras.

A análise da produtividade média do café nas microrregiões mineiras ratificou, primeiramente por meio da utilização de técnicas de Aede, e depois da econometria espacial, a hipótese de dependência espacial entre as distintas unidades, mostrando que as técnicas de econometria convencional não são as mais indicadas para esse tipo de análise.

Pode-se constatar que existe uma forte influência espacial na produtividade média do café (formação de clusters), isto é, a produtividade de uma determinada microrregião sofre influência da produtividade em localidades vizinhas e vice-versa. Além disso, a estatística Lisa sugere uma reestruturação da produtividade cafeeira entre as microrregiões mineiras durante o período 1997-2006.

Por sua vez, com relação à hipótese de homogeneização da produtividade média do café, o fenômeno da $\beta$ convergência mostra-se extremamente significativo. Todos os modelos expostos no trabalho apresentaram um coeficiente $\beta$ negativo, o que revela que as microrregiões com menor produtividade média do café em 1997 tenderam a se aproximar daquelas com maior produtividade média em um decênio e a uma velocidade acelerada, conforme indica a taxa de convergência de $7,6 \%$ anuais.

Entre todos os modelos de convergência, o modelo de erro espacial foi o que melhor explicou esse fenômeno entre as microrregiões mineiras, conclusão corroborada pelos significativos coeficientes $\alpha, \beta$ e $\lambda$ e pelas estatísticas AIC, SC e LIK.

Um fenômeno que poderia explicar esse deslocamento do centro dinâmico da produtividade média do café em Minas Gerais é o crescente aumento das plantações de cana-de-açúcar, soja, trigo e milho nas microrregiões mineiras, que fazem fronteira com os estados de São Paulo e Goiás. Nesse sentido, o agronegócio intensivo em capital, voltado para a produção de etanol e grãos, dada a sua extraordinária rentabilidade, parece estar contribuindo de maneira decisiva para aumentar a produtividade do café nas microrregiões mineiras mais pobres. 


\section{Referências}

ALMEIDA, E. S.; PEROBELLI, F. S.; FERREIRA, P. G. C. Existe convergência espacial da produtividade agrícola no Brasil? Revista de Economia e Sociologia Rural, v. 46, n. 1, p. 31-52, jan./mar. 2008.

ALMEIDA, E. S; HADDAD, E. A; HEWINGS, G. J. D. The spatial pattern of crime in Minas Gerais: an exploratory analysis. Economia Aplicada, v. 9, n. 1, p. 39-55, 2005.

ANSELIN, L. Exploring Spatial Data with GeoDa: a workbook. Urbana-Champaign: University of Illinois, 2005.

. Interactive tecniques and exploratory spatial data analysis. In: LONGLEY P. A. et al. (Ed.). Geographical information system: principles, techniques, management and applications. Wiley: New York. 1998. p. 253-365.

. Local indicators of spatial association - LISA. Geographical Analysis, v. 27, n. 2, p. 93-115, Apr. 1995. Texas, 1999.

Spatial econometrics. Dallas: Bruton Center: School of Social Sciences, University of

. Spatial econometrics: methods and models. Boston: Kluwer Academic, 1988.

ANSELIN, L.; BERA, A. Spatial dependence in linear regression models with an introduction to spatial econometrics. In: ULLAH A.; GILES, D. E. (Ed.). Handbook of applied economic statistics. New York: Marcel Dekker, 1998. p. 237-289.

BANCO DE DESENVOLVIMENTO DE MINAS GERAIS. Minas Gerais do Século XXI. Belo Horizonte: Rona, 2002.

BARRO, R. J.; SALA-I-MARTIN, X. Convergence across states and regions. Brookings Papers, Economic Activity, n. 1, p. 107-182, 1991.

. Convergence. Journal of Political Economy, v. 100, n. 2, p. 223-251, 1992.

BAUMOL, W. J. Productivity growth, convergency, and welfare: What the long run data show. American Economic Review, v. 76, n. 5, p. 1072-85, 1986.

BRASIL. Ministério da Agricultura Pecuária e Abastecimento. Fundo de Defesa da Economia Cafeeira: Funcafé 2007. (Relatório de Atividades). Disponível em: <http://www.agricultura. gov.br/arq_editor/file/3406_relatorio_de_atividades_do_funcafe_2007.pdf $>$. Acesso em: 2 dez. 2009.

CLIFF, A. D.; ORD, J. K. Spatial processes: models and applications. London: Pion, 1981.

FLORAX, R. J. G. M.; FOLMER, H.; REY, S. J. Specification searches in spatial econometrics: the relevance of Hendry's methodology. Regional Science and Urban Economics, v. 33, n. 5, p. 557-79, 2003.

IBGE. Sistema IBGE de Recuperação Automática. Disponível em: < http://www.sidra.ibge.gov. br/bda >. Acesso em: 5 jul. 2008. 
KELEJIAN, H. H.; PRUCHA, I. R. A. Generalized moments estimator for the autoregressive parameter in a spatial model. International Economic Review, v. 40, n. 2, p. 509-533, 1999.

LE GALLO, J.; ERTHUR, C. Exploratory spatial data analysis of the distribution of regional per capita. GDP in Europe, 1980-1995. Papers in Regional Science, v. 82, n. 2, p. 175-201, 2003.

LOPES, J. L. Avaliação do processo de convergência da produtividade da terra na agricultura brasileira no período 1960 a 2001. 2004. 193 f. Tese (Doutorado em Ciências) - Escola Superior de Agricultura Luiz de Queiroz, Universidade de São Paulo, Piracicaba, SP, 2004.

OLIVEIRA, A. A. S. et al. Estrutura e dinâmica da cafeicultura em Minas Gerais. Revista de Economia, v. 34, n. 1, p. 121-142, 2008.

PEROBELLI, F. S. et al. Análise espacial da produtividade do setor agrícola brasileiro: 19912003. Nova Economia, v. 17, n. 1, p. 65-95, 2007.

PEROBELLI, F. S.; FARIA, W. R.; FERREIRA, P. G. C. Análise da convergência espacial do PIB per capita no estado de Minas Gerais. Revista Brasileira de Estudos Regionais e Urbanos, v. 1, n. 1, p. 85-113, 2009.

PIMENTEL, E.; HADDAD, E. A. Análise da distribuição espacial da renda no estado de Minas Gerais: uma abordagem setorial. São Paulo: Nereus, 2004. (Texto para discussão, n. 02-2004).

QUAH, D. Regional convergence clusters across Europe. European Economic Review, v. 40, n. 3, p. 951-958, 1996.

REY, J. S.; MONTOURY, B. D. US Regional income convergence: a spatial econometric perspective. Regional Studies, v. 33, n. 2, p. 143-156, 1999.

SANTOS, V. E. et al. Análise do setor e processamento de café em Minas Gerais: uma abordagem matriz insumo-produto. Revista de Economia e Sociologia Rural, Piracicaba, v. 47, n. 2, p. 363-388, 2009.

SOUZA, M. C.; PEROBELLI, F. S. Análise da distribuição territorial da sojicultura no Brasil: 1991-2003. Revista Econômica do Nordeste, v. 39, n. 1, p. 46-65, 2008.

SOUZA, R. M.; PEROBELLI, F. S. Diagnóstico espacial da concentração produtiva do café no Brasil, no período de 1991 a 2003. Revista de Economia e Agronegócio, v. 5, n. 3, p. 353377, 2007.

VALE, S. M. L. R.; TEIXEIRA, M. B.; SANT'ANNA, J. C. O. Estilos gerenciais e eficácia administrativa na produção de café. Revista de Economia e Agronegócio, Viçosa, v. 4, n. 4, p. 533-552, 2006.

Recebido em: 07/02/2012.

Acesso em: 25/04/2014. 